\title{
High Time Resolution Observations of the Crab Pulsar with the UCL MIC Detector
}

\author{
R. Much \\ Astrophysics Division, Space Science Department of ESA, P.O. Box \\ 299, 2200 AG Noordwijk, Netherlands
}

A. Carramiñana

Instituto Nacional de Astrofísica, Optica y Electroníca, Luis Enrique Erro 1, Tonantzintla, Puebla 72840, Mexico

J.L.A. Fordham

Department of Physics 83 Astronomy, University College London, Gower Street, London WC1E 6BT, UK

H. Kawakami

Mullard Space Science Laboratory, University College London, Holmbury St. Mary, Dorking, Surrey, RH5 6NT, UK

R. Michel

Instituto de Astronomia, Observatorio Astronomico Nacional, Apartado Postal 877, Codigo Postal 22860, Ensenada B.C., Mexico

\begin{abstract}
High time resolution $(41.4 \mu \mathrm{s})$ observations of the Crab Pulsar have been undertaken on the $2.1 \mathrm{~m}$ telescopes at San Pedro Martir and the Observatorio Astrofisico Guillermo Haro (MX) in January/February 1999. In our prelimenary analysis of the Crab Pulsar's light curve we obtained a slightly narrow widths on the seondary pulse than that reported by Percival et al. Our data indicate the presence of substructures in the pulsar's main pulse, though not statistically convincing.
\end{abstract}

\section{Observation}

Time resolved spectroscopic data on the Crab Pulsar was obtained during observing trials with the UCL MIC photon counting detector operating in a novel, high time resolution, mode called Freerun (Fordham et al. 1999). Here we report on the preliminary analysis of the pulsar light curve obtained by collapsing in dispersion direction the spectroscopic data from the San Pedro Martir observations. The detector was mounted behind a Boller \& Chivens spectrograph and the observational waveband extended from $3800 \mathrm{~A}$ to $6500 \mathrm{~A}$. Due to weather conditions useful data could only be acquired on three nights (Jan 22 to 24) resulting in a total of $12 \times 10^{6}$ photons being accumulated, where $1.8 \times 10^{6}$ photons are attributed to the pulsar. 


\section{Analysis, Results and Outlook}

Our analysis of the Crab light curve followed the procedure described in Percival et al. (1993), i.e. the separation between, and the widths of the main and secondary pulses were determined from the autocorrelation function (ACF) and from a polynomial fit.

Table 1. Crab light curve analysis, as derived from ACF and polynomial fits to the data. Uncertainties are estimated as \pm 0.0013 duty cycle and \pm 0.0021 duty cycle for individual night and all data respectively.

\begin{tabular}{|c|c|c|c|c|c|c|}
\hline Jan & \multicolumn{2}{|c|}{ ACF Width } & Peak & \multicolumn{2}{|c|}{ Fit Width (FWHM) } & Peak \\
\hline 1999 & Main & Secondary & Separation & Main & Secondary & Separation \\
\hline all & 0.0433 & 0.0902 & 0.4154 & 0.0468 & 0.0878 & 0.4084 \\
\hline 22 & 0.0420 & 0.0902 & 0.4154 & 0.0433 & 0.0816 & 0.4086 \\
\hline 23 & 0.0433 & 0.0915 & 0.4141 & 0.0445 & 0.0828 & 0.4077 \\
\hline 24 & 0.0433 & 0.0902 & 0.4141 & 0.0457 & 0.0841 & 0.4094 \\
\hline
\end{tabular}

The peak widths become broader when combining all exposures. This can be explained by a residual phase shift between exposures for the different nights. The results from our ACF analysis are, in general, smaller than those obtained by Percival et al. (1993). However the phase separation between the two peaks and the width of the main pulse are, within the uncertainties, in agreement with Percival's findings. The width of the secondary pulse is found to be narrower in our data, this difference being enhanced when fitting with a polynomial. We obtained for the ratio of the maxima of the main to secondary peak $3.90 \pm 0.10$, which agrees with the number reported by Percival et al. (3.78 00.11 ).

While the light curve resulting from summation of all exposures looks smooth, a close visual inspection of the sequence of individual light curves (1000s exposures) obtained in the course of one night suggests the presence of systematic substructures persisting over several exposures. However, comparing profiles of the main pulses (area with best statistics) of the individual light curves provided no significant evidence for the profiles not being associated with the same parent distributions. The profiles for the three nights are drawn with a probability of $0.54 \pm 0.11,0.46 \pm 0.11$ and $0.52 \pm 0.11$ from the same parent distributions.

The observing trials were primarily related to obtaining phased locked spectra of the Crab pulsar. However, insufficient signal was obtained to allow a spectroscopic analysis and hence an analysis of the light curve has been concentrated upon. New observations, with a now proven technology, are planned for obtaining further spectral information.

Acknowledgments. The authors thank Andrew Lyne, Dick Manchester and David Nice for explaining the intricacies of the TEMPO software used for enabling detector synchronization with the pulsar.

\section{References}

Fordham, J. et al. 1999, these proceedings

Percival, J.W. et al. 1993, APJ, 407, 276 